\title{
Mujer y juego online. Propuesta de tratamiento de un caso de adicción a video bingo
}

\section{Woman and online gambling. A case study of addiction to video bingo}

Fecha de recepción: 13-02-2019

Fecha de aceptación: 23-05-2019

\author{
Marta Marcos \\ Psicóloga. Universidad de València. Unidad de Investigación \\ "Juego y adicciones tecnológicas". \\ Departamento de Psicología Básica \\ Mariano Chóliz \\ Profesor. Universidad de València. Unidad de Investigación \\ "Juego y adicciones tecnológicas". \\ Departamento de Psicología Básica.
}

\section{resumen/ahstract:}

Desde la legalización del juego online en España el trastorno de juego se ha incrementado notablemente, ya que ha aumentado la disponibilidad y accesibilidad de un tipo de juego que es, de por sí, más adictivo que el tradicional. Aunque las tasas de prevalencia son superiores en hombres que en mujeres, ellas no son ajenas a este problema, que también suele concurrir con otros problemas de índole emocional.

El trabajo que presentamos es un estudio de caso de una mujer procedente de los servicios de salud mental, que padecía, además de adicción al juego, una importante sintomatología clínica. Se describe el protocolo de tratamiento de adicción al juego online desarrollado en la Unidad de Investigación: "Juego y adicciones tecnológicas" de la Universidad de Valencia, que recoge las aportaciones de diversas investigaciones científicas. El tratamiento conductual cognitivo que le fue aplicado no solamente tuvo eficacia en la reducción del juego, sino que produjo un radical cambio en estilo de vida, actitudes hacia el juego y sintomatología clínica.

Since the legalization of online gambling in Spain, gambling disorder has increased significantly, due to the increased availability and accessibility of a type of game that is, in itself, more addictive than the traditional one. Although the prevalence rates are higher in men than in women, they are not alien to this problem, which also tends to concur with other emotional problems.

This paper is a case study of a woman sent from the mental health services, who suffered, in addition to gambling addiction, an important clinical symptomatology. We describe the online gambling addiction treatment protocol developed in the Research Unit: "Game and technological addictions" of the University of Valencia, which collects the contributions of several scientific researches. The cognitive behavioral treatment that was applied not only had efficacy in the reduction of gambling, but also produced a radical change in lifestyle, attitudes towards gambling and clinical symptomatology.

\section{palabras clave/keywords:}

Trastorno de juego; trastorno de juego online; adicción al juego, género; tratamiento conductual cognitivo Gambling disorder; online gambling disorder; gambling addiction; gender; cognitive behavior therapy 
La mayoría de los estudios reflejan que los hombres juegan más que las mujeres a juegos de apuestas, envite y azar (Chóliz, Marcos y Lázaro-Mateo, 2019; Wardle et al., 2007; Welte, Barnes, Wieczorek, Tidwell y Parker, 2002) y también muestran tasas de prevalencia de juego patológico más elevadas (Abbot, Volberg y Ronberg, 2004; Bakken, Götestam, Grawe, Wenzel y Øren, 2009; Blanco, Hasin, Petry, Stinson y Grant, 2006; Chóliz, Marcos y Lázaro-Mateo, 2019; Griffiths y Barnes, 2008; Johansson, Grant, Kim, Odlaug y Gotestam, 2009; Volberg, 2001; Wardle et al., 2007). A pesar de ello, es necesario hacer varias precisiones al respecto.

En primer lugar, habría que indicar que esto es cierto para la mayoría de los juegos de azar, pero no para todos, ya que juegos como las loterías presentan unas tasas de prevalencia similares entre hombres y mujeres (Abbott, Volberg y Ronnberg, 2004), lo cual justifica el hecho de que es necesario distinguir entre los diferentes juegos cuando se aborda el efecto del juego, así como las motivaciones para jugar. Por otro lado, los estudios clínicos manifiestan que, si bien las mujeres tienen menores tasas de adicción al juego que los hombres, también acuden a recursos asistenciales en una proporción mucho menor de la que les correspondería. En un estudio realizado con 1.277 jugadores patológicos en tratamiento (Chóliz, 2016) solamente el 6,4\% de los pacientes eran mujeres, frente al 93,6\% de hombres, lo cual es una cifra considerablemente más baja que la que correspondería de acuerdo con la tasa de prevalencia que representa este trastorno en mujeres que, se sitúa en torno al 22\% (Chóliz, Marcos y Lázaro-Mateo, 2019). Ello indica que las mujeres acuden a tratamiento en una proporción que no solamente es menor que los hombres, sino que es más baja de lo que representa, lo cual indica que este tema de género debe abordarse desde una perspectiva científica. En tercer lugar, las propias características de las nuevas modalidades de juego online, de reciente legalización en España, también muestran unas diferencias notables en función del género, ya que solamente el 2,4\% de las mujeres han jugado alguna vez a juegos online, frente al 10\% de los hombres (Chóliz, Marcos y Lázaro-Mateo, 2019), diferencias que también se observan en otros estudios (Griffiths y Barnes, 2008; Griffiths, Wardle, Orford, Sproston y Erens, 2009). Por último, las mujeres suelen comenzar a jugar más tarde que los hombres, pero la progresión para desarrollar juego patológico es más rápida (Granero et al., 2009) y el pronóstico es peor que el de los hombres, probablemente porque el juego en las mujeres está relacionado en mayor medida que en los hombres con la superación de malestar emocional, lo cual es un hecho que obliga a abordar el tema del género en la adicción al juego desde una perspectiva científica.

El caso que se presenta en este trabajo es el de una mujer con problemas de juego patológico con videobingo, que es una de las nuevas modalidades de juego online, en concreto, bingo a través de Internet. Se describen las principales características del caso, así como el protocolo de tratamiento aplicado desarrollado por los autores para el tratamiento de la adicción al juego online.

\section{MÉTODO}

\section{CASO CLínICO}

Contextualización sociodemográfica y motivo de consulta. Paciente C. mujer de 38 años de edad, con pareja y madre soltera de una niña de 11 años. De profesión administrativa, ac- 
tualmente se encuentra en paro. Acude a consulta derivada del centro de salud mental de su zona por problema con el juego online, concretamente, videobingo a través de su smartphone. La paciente manifiesta su queja principal refiriendo que "resulta insostenible seguir con esto. Se te pasan las horas y dejas de hacer todo tipo de cosas". Atribuye su problema "al puro aburrimiento". Se muestra abatida, triste y con llanto continuo. Actualmente recibe tratamiento en psiquiatría por trastorno de ansiedad y depresión: Pristiq 50-100mg, Apaloz, Dobupal, Lexatin, transilium $10 \mathrm{mg}$, lormetazepam $10 \mathrm{mg}$ y Noctamid.

En el primer contacto C. firmó el consentimiento informado y se le explicó el compromiso del terapeuta en base al Código Deontológico del Psicólogo, resaltando la confidencialidad de la información y el secreto profesional.

Antecedentes. La paciente refiere episodios de impulsividad en la adolescencia que la llevaban a "arrancar puertas y lanzar cosas". Refiere haber tenido otro episodio de juego online anterior al momento de quedarse embarazada de su hija, sin embargo, no ha recibido ningún tratamiento anterior a causa de este problema. Niega haber consumido drogas y recientemente ha tenido problemas para mantenerse en su empleo debido a su sintomatología ansiosa y depresiva, lo cual le condujo a solicitar la baja laboral y más tarde sería despedida. Ha sufrido malos tratos por parte de su expareja. Respecto a la familia apenas nombra a su padre, mientras que a su madre la ve como una persona poco afectiva aunque reconoce su ayuda en todo este problema. Con la hermana no mantiene relación y no se llevan bien debido a que esta fue quien contó al padre su problema con el juego a consecuencia de que C. llamara al padre para pedirle dinero prestado para jugar. No existen antecedentes familiares con problemas por juego, aunque su hermana se encuentra en tratamiento por depresión en el momento de la llegada a consulta. Su pareja actual es para C. su único amigo y su apoyo principal.

Historia de Juego. C. comenzó a jugar a juego online en 2007, a la edad de 27 años, concretamente a video bingo a través de páginas ilegales, ya que el juego online no se legaliza hasta el 2011. Este primer episodio de juego coincidió con la pérdida del trabajo y la posterior lesión en una pierna que le llevaron a tener que estar en reposo y por mero aburrimiento se inició en el mundo del juego de apuestas por Internet. La frecuencia de juego era diaria, si bien no recuerda qué cantidades jugaba. Al quedarse embarazada, el problema desapareció sin necesidad de ayuda, pues “encontró motivos más importantes que el juego". Pasó 10 años sin jugar y coincidiendo con un nuevo despido de su trabajo de teleoperadora y el cobro de la indemnización, se inició de nuevo a jugar a bingo online. En este segundo episodio de juego, pasa el día sin salir, no tiene interés por nada y aumenta $20 \mathrm{~kg}$. de peso a causa de su inexistente actividad y estilo de vida sedentario. Apenas cumple con llevar a su hija al colegio. El dinero que gasta es todo el que percibe de la prestación que cobra por desempleo, siendo la madre de C. quien sufraga sus gastos y los de su hija. Las cantidades de dinero perdidas y las deudas son el detonante que hizo que reconociera que tenía un problema y lo contara a su pareja. Acudió al médico de atención primaria, quien le remitió al centro de salud mental de la zona para tratamiento. Respecto a las áreas más perjudicadas a consecuencia del juego, destacó la economía, seguida de las relaciones sociales y las actividades de ocio. 
El problema actual de juego se inició a principios de 2018. El patrón de frecuencia de juego era diario y en horario indistinto, ya fuera mañana, tarde o noche. La cuantía de cada partida era de $4 €$ que correspondían a la apuesta de 4 cartones de $0,50 €$ cada vez. La duración solía ser de horas y hasta que se le terminaba el dinero de la prestación mensual (aprox. $450 €)$. En ocasiones ha llegado a pedir algún microcrédito.

Diseño. Según la clasificación de Montero y León (2005) se empleó un diseño de caso único AB (Barlow y Hersen, 1988), siendo la intervención de carácter individual y grupal con seguimientos (1-3-12-24 meses) después del alta terapéutica.

\section{Análisis del caso.}

En el momento inicial de la entrevista refirió estar jugando menos, aunque las deudas de C. ya ascendían a $800 €$ a causa de los microcréditos. Refirió tener la cuenta "intervenida" ya que su madre estaba autorizada. A pesar de esto, reconoció que seguía jugando y que cuando lo hacía "le daba todo exactamente igual". La última vez que jugó fue la semana anterior y gastó $600 €$ en unas horas. Como resultado aparece en C. la culpabilidad aunque indica que lo hace al día siguiente de jugar: "es como si no estuviera pensando en el mañana". Se siente mal por perder dinero, mentir, ocultar el juego y aislarse de los demás hasta el punto de no coger ni el teléfono cuando le llaman. Situación que agrava aún más el problema de juego y que hace que "escape" de ella volviendo a jugar, sobre todo en los momentos en los que no hace nada, se tumba y está sola. Respecto a la interferencia en el trabajo, no ha habido juego mientras ha estado en activo. En casa se encierra y aísla para jugar, refiere mal humor con su hija y ha dejado de hacer cosas cotidianas como hacer comidas y dedicar tiempo compartido con la hija. No tiene interés por nada y se encuentra muy deprimida. Pasa la mayor parte de día tumbada y apenas cumple con llevar a la hija al colegio. Refiere que la madre se encuentra muy preocupada porque ve su problema como una enfermedad, pero que su pareja, aunque está preocupado, confía más en ella porque ve su problema como un error causado por estar sin hacer nada. C. reconoce que el juego "se le ha ido de las manos", pues juega hasta que el dinero se le acaba y si le toca un premio, tampoco es capaz de parar y retirarse.

En cuanto al análisis funcional de la conducta problema (O’Neill, Homer, Albin, Storey y Sprague, 1990), los estímulos antecedentes de la conducta de juego eran: de tipo ambiental o externos como disponer de dinero el décimo día de cada mes, que es cuando cobraba la prestación, estar sin trabajar ni tener nada que hacer y cuando llevaba tiempo jugando y no le había salido un premio; e internos como estar mal anímicamente, ver muy complejo el futuro y sentirse sola. Las conductas problema eran principalmente motoras y cognitivas. En cuanto a las primeras, apostaba una y otra vez hasta quedar sin dinero, cuando le tocaba un premio realizaba verbalizaciones de alegría y euforia y aumentaba la rapidez de las pulsaciones y el botón de inicio de partidas nuevas. En cuanto a las segundas, aparecían pensamientos de estar en racha, con creencia firme de que le tocaría acompañada de cuasidisociación que le hacían sentirse otra. Existían fuentes de refuerzo positivo al ponerse a jugar con su móvil, era un momento de distracción y entretenimiento que, además, esporádicamente y a modo de refuerzo intermitente parcial, se conjugaba con algún premio. Sin embargo, la consecuencia más importante que contribuía a mantener el problema era el ali- 
vio que encontraba a estar sin hacer nada, aburrida y con sentimientos de profunda tristeza y soledad que le provocaban un enorme malestar emocional. Para C. la principal motivación para apostar era la "evasión" de las propias emociones negativas. Así, el problema se mantenía por refuerzo negativo que aliviaba a corto plazo su malestar, pero que perpetuaba a largo plazo la conducta de juego patológico.

\section{Instrumentos de evaluación.}

Tras la entrevista inicial y el cuestionario de historia de juego, se aplicó una batería de evaluación psicológica de amplio espectro que incluía medidas de aquellas variables más directamente relacionadas con el riesgo y el desarrollo de problemas de juego y adicción al juego. Se incluyó una batería de pruebas en las que se evaluó el estado emocional, síntomas clínicos y psicopatológicos, procesos implicados (impulsividad, actitudes hacia el dinero) y consumo de sustancias, entre otras. En este estudio se analizan los resultados de las siguientes pruebas y autoinformes:

- Patrón de juego encuesta Ludens (Chóliz, 2017). Se trata de un registro de la frecuencia de los diferentes tipos de juego presentes en España, tanto tradicionales como online.

- Autorregistro de juego y actividades alternativas. Consiste en el análisis funcional de la conducta de juego y las actividades alternativas.

• NODS (Gerstein, Hoffmann, Larison, Engelman, Murphy, Palmer, ... y Hill, 1999). Entrevista utilizada para el diagnóstico de la adicción al juego según los criterios del DSM-5.

- Síntomas clínicos y psicopatológicos, SCL-90 (Derogatis, 1979). Se trata de una escala que evalúa nueve síntomas clínicos y tres indicadores de malestar. Las dimensiones sintomatológicas primarias son Somatización, Obsesividad-compulsividad, Sensibilidad interpersonal, Depresión, Ansiedad, Hostilidad, Ansiedad fóbica y Psicoticismo. Los tres índices globales que proporcionan una medida de distrés son los siguientes: Índice de Sintomatología General (GSI, General Symptom Index), Índice de Alteración de Síntomas Positivos (PSDI, Positive Symptom Distress Index) y el Total de Síntomas Positivos (PST, Positive Symptom Total).

Para registrar la línea base sobre el juego se empleó un autorregistro con columnas en relación a: fecha y hora, tipo de juego y lugar, consumo de sustancias (antes, durante y después), dinero gastado/ganado, tiempo dedicado, qué estabas haciendo antes, qué pensabas/ sentías antes, qué hiciste después, qué pensaste/sentiste después.

Tras esta evaluación inicial, se realizó un diagnóstico de C. de juego patológico grave, teniendo en cuenta los resultados del NODS en base a los criterios diagnósticos de Juego Patológico según el Manual Diagnóstico y Estadístico de los Trastornos Mentales, DSM-5 (APA, 2013) para el comportamiento de juego desadaptativo, persistente y recurrente, con más de cuatro de los siguientes: preocupación por el juego, necesidad de apostar cantidades crecientes de dinero, fracaso repetido del esfuerzo por controlar, interrumpir o detener el juego, inquietud/irritabilidad si intenta interrumpir o detener el juego, el juego se utiliza 
como estrategia para escapar de los problemas o aliviar la disforia, tras la pérdida del dinero se vuelve a jugar para recuperar, se engaña a los miembros de la familia, terapeutas u otros sobre el grado de implicación en el juego, se han arriesgado o perdido relaciones interpersonales, trabajo u oportunidades significativas debido al juego, se confía en que los demás se hagan cargo de las deudas contraídas por el juego para alivio de la desesperada situación financiera; además, se ha de descartar que el comportamiento de juego no es debido a un episodio maníaco.

La intervención llevada a cabo se basó en el protocolo de tratamiento para la adicción al juego online (Chóliz y Marcos, 2019) que se describe a continuación.

\section{Tratamiento.}

La intervención constó de 19 sesiones (15 individuales y 4 grupales) con periodicidad semanal y duración aproximada de una hora, además de las correspondientes a seguimientos al mes y a los tres meses. En el momento de escribir el trabajo quedan por analizar el estado de la paciente a los 6, 12 y 24 meses de seguimiento. El seguimiento consiste en una breve visita (de aproximadamente 15-20 minutos) en la cual la paciente indique, entre otros aspectos: cómo se encuentra; si ha tenido algún episodio de juego; si tiene o ha tenido deseos de jugar; cómo los ha superado en el caso de que los haya tenido; si mantiene el estilo de vida alternativo, así como cualquier otro aspecto que considere relevante indicar.

El tratamiento se estructuró en las siguientes fases:

Fase 1. Análisis, evaluación y diagnóstico. Centrada en la evaluación del problema, se llevaron a cabo cuatro sesiones de entrevista psicológica y se solicitaron la cumplimentación de diferentes cuestionarios, la historia de juego y autorregistro de juego, con el fin de tomar la línea base. Desde la motivación para el cambio como un estilo de relación entre el paciente y el terapeuta determinada por la entrevista motivacional (EM) (Miller y Rollnick, 1991, 2002), durante estas cuatro primeras sesiones, además de ofrecer mucha contención y generar el vínculo dentro un clima de empatía y cordialidad exento de juicios, se dio información acerca del proceso adictivo y cómo estaban diseñadas las características de los propios juegos. Se realizó el encuadre y la firma del contrato terapéutico que recogió el compromiso de C. sobre las pautas a seguir para llevar a cabo la intervención. Las tareas para casa solicitadas fueron la cumplimentación del registro de línea base de la conducta de juego, balance decisional y la puesta en marcha del diario de actividades realizado en consulta a modo de terapia de activación.

Fase 2. Abstinencia y superación del malestar. El objetivo de esta segunda fase en la que se dedicaron cuatro sesiones se centró en que C. consiguiera la abstinencia de juego y la superación del malestar provocado por la privación del juego o las deudas contraídas. Se realizó un plan de pagos sobre la deuda pendiente de microcréditos en base a los ingresos mensuales de C., quien debía tomar las riendas del problema y gestionar y acordar telefónicamente los pagos a devolver que se establecieron previamente en sesión. Asimismo, en presencia de la madre, que es quien ejerció como coterapeuta en esta fase, se explicaron las pautas a seguir para el control de estímulo y C. accedió a pasar el control del dinero y las tarjetas a la madre, comprometiéndose a llevar únicamente dinero de bolsillo para gastos 


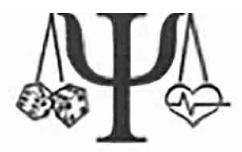

Juego Ético



Figura 1: Modelo de contrato terapéutico. 
diarios que debería justificar mediante tickets. Se comenzó el entrenamiento en técnicas de reducción del malestar: relajación muscular progresiva de Jacobson (RMP), entrenamiento en técnicas de respiración y se continuó con el horario de actividades diarias muy estructuradas con el fin de promover la realización de conductas alternativas, que sirvieran a su vez para generar un estilo de vida posteriormente.

Se comenzó pautando un paseo por las mañanas cuando dejara a la hija en el colegio y se continuó con el establecimiento de tareas cotidianas, tales como realizar la compra y encargarse de elaborar las comidas para la familia todos los días. El tiempo de la tarde se estructuró principalmente con su hija: recogida del colegio, ayuda en sus deberes y ocio compartido. Con el objetivo de fomentar el apoyo social de C. y socializar con otras madres, se sugirió bajar al parque donde estarían las compañeras del colegio de la hija. Las tareas para casa que se solicitaron fueron las siguientes: cumplimentación y presentación del formulario de autoexclusión, cancelación de sus datos personales de todas las páginas de juego online en las que había jugado mediante correo postal, cumplimentación y presentación online del fichero de auto protegidos de microcréditos, autorregistro de deseo de juego y registro de actividades alternativas de ocio y puesta en práctica de las técnicas entrenadas para reducir la ansiedad motivando a $\mathrm{C}$. para la práctica en casa a diario con el fin de conseguir que la relajación formara parte de sus actividades diarias. Por esta razón, antes de cada sesión, se reforzó cada aspecto que resaltó C. sobre los beneficios de practicar las técnicas entrenadas. También se le propuso un calendario donde se apuntó la fecha en que abandona el juego. Con la finalidad de aprender a autorreforzarse adaptativamente, debía pensar en algo cuyo valor fuera gratificante y extraordinario y que recibiría al cumplimiento de un mes abstinente. C. decidió reforzar la abstinencia continuada con una cena con su actual pareja y otra pareja de amigos en un buen restaurante de Valencia.

Tabla 1: Ejemplo de registro de actividades de C. durante el tratamiento.

\begin{tabular}{|c|c|c|c|c|c|c|c|}
\hline & LUNES & MARTES & MIÉRCOLES & JUEVES & VIERNES & SÁBADO & DOMINGO \\
\hline \multirow{4}{*}{ MAÑANA } & \multicolumn{7}{|c|}{ Desayunar } \\
\hline & \multicolumn{7}{|c|}{ Llevar a Claudia al colegio } \\
\hline & $\begin{array}{l}\text { Entrevista } \\
\text { de trabajo }\end{array}$ & Terapia & Comprar & $\begin{array}{l}\text { Entrevista } \\
\text { de trabajo }\end{array}$ & Gimnasio & Comprar & $\begin{array}{l}\text { Recoger } \\
\text { Claudia }\end{array}$ \\
\hline & & & & & & & Pasear \\
\hline \multirow[t]{2}{*}{ TARDE } & \multicolumn{7}{|c|}{ Hacer la comida // Comer } \\
\hline & $\begin{array}{c}\text { Curso de } \\
\text { trabajo }\end{array}$ & & $\begin{array}{l}\text { Ayudar a } \\
\text { Claudia con } \\
\text { deberes }\end{array}$ & $\begin{array}{l}\text { Entrevista } \\
\text { de trabajo }\end{array}$ & $\begin{array}{c}\text { Ocio } \\
\text { compartido } \\
\text { sobrinos }\end{array}$ & $\begin{array}{c}\text { Llevar } \\
\text { Claudia } \\
\text { fiesta }\end{array}$ & $\begin{array}{c}\text { Ocio } \\
\text { compartido } \\
\text { con Claudia }\end{array}$ \\
\hline NOCHE & \multicolumn{7}{|c|}{ Hacer la cena // Cenar // Dormir } \\
\hline
\end{tabular}


Tabla 2: Ejemplo de autorregistro de deseo de juego durante el tratamiento de C.

\begin{tabular}{|c|c|c|c|c|c|c|c|c|c|c|c|}
\hline Fecha y Hora & $\begin{array}{c}\text { Intensidad } \\
\text { del deseo de } \\
\text { jugar } \\
(1-10)\end{array}$ & $\begin{array}{c}\text { Consumo de } \\
\text { sustancias } \\
(\mathrm{A}, \mathrm{Du}, \mathrm{De})\end{array}$ & $\begin{array}{l}\text { Qué estabas } \\
\text { haciendo } \\
\text { ANTES }\end{array}$ & $\begin{array}{c}\text { Qué estabas } \\
\text { pensando } \\
\text { ANTES }\end{array}$ & $\begin{array}{c}\text { Señal que inició } \\
\text { el deseo } \\
(I / E x)\end{array}$ & $\begin{array}{l}\text { Sintomas } \\
\text { que } \\
\text { notaste }\end{array}$ & $\begin{array}{c}\text { Capacidad } \\
\text { de resistir el } \\
\text { impulso } \\
(1-10)\end{array}$ & $\begin{array}{c}\text { Qué } \\
\text { hiciste } \\
\text { DESPUÉS } \\
\text { (afront.) }\end{array}$ & $\begin{array}{l}\text { Qué pensaste } \\
\text { DESPUÉS } \\
\text { (afront.) }\end{array}$ & $\begin{array}{l}\text { ¿Has } \\
\text { jugado? }\end{array}$ & $\begin{array}{l}\text { ¿Que podrias haber } \\
\text { hecho para no jugar? }\end{array}$ \\
\hline Lunes, 16h & 10 & & $\begin{array}{l}\text { Tumbada, } \\
\text { viendo TV }\end{array}$ & & Publicidad & Ansiedad & 10 & Levantarme & Orgullo & NO & \\
\hline $\begin{array}{l}\text { Martes } \\
9: 30 \mathrm{~h}\end{array}$ & 10 & & Tumbada & & Recuerdo & Ansiedad & 10 & Levantarme & Orgullo & NO & \\
\hline $\begin{array}{l}\text { Miércoles } \\
\text { 11:30h }\end{array}$ & 10 & & Paseo & & Recuerdo & Ansiedad & 10 & $\begin{array}{l}\text { Seguir } \\
\text { andando }\end{array}$ & Depresión & NO & \\
\hline $\begin{array}{l}\text { Jueves } \\
9: 30 \mathrm{~h}\end{array}$ & 10 & & Compra & & Recuerdo & Ansiedad & 10 & $\begin{array}{c}\text { Seguir } \\
\text { comprando }\end{array}$ & Depresión & NO & \\
\hline
\end{tabular}

Fase 3. Descondicionamiento y estilo nuevo de vida. Los objetivos que se persiguieron en esta tercera fase compuesta por seis sesiones fueron descondicionar las claves y consecuencias del juego, así como disminuir el interés y el agrado que provoca y la adquisición de un estilo de vida saludable. Para conseguirlo se siguió el procedimiento desarrollado por Echeburúa y Fernández Montalvo (2005) consistente en exposición en vivo con prevención de respuesta graduada en base a unidades subjetivas de síndrome de abstinencia (USSA), así como el entrenamiento en estrategias de afrontamiento que consiguieran que $\mathrm{C}$. evolucionara de ser una persona autorreferente a ser una persona autoeficaz. Las técnicas que se utilizaron fueron: relajación, detención de pensamiento, autoinstrucciones, balance decisional, solución de problemas, detención de la respuesta y control del impulso. Para modelar la exposición en consulta, se llevó a cabo el entrenamiento en contexto terapéutico y después se mandó que hiciera en casa tareas similares dos veces al día. Sesión tras sesión, se fue complicando más la exposición de forma que fuera progresivamente parecida a la situación real de juego que realizaba habitualmente. En esta fase ejerció como coterapeuta su pareja.

La graduación de la dificultad de los ejercicios se realizó de la siguiente manera y según tres niveles:

Nivel 1: La pauta fue realizar el ejercicio de exposición dos veces al día (mañana y tarde). Para ello debería navegar desde la tablet y no el smartphone, tal y como solía hacer cuando jugaba a video bingo, al tiempo que debía hacerlo sentada, de forma contraria a la habitual (que era tumbada en su cama). En este primer nivel más básico de dificultad se sugirió a C. que entrara a navegar por páginas de bingo online que no eran las que frecuentaba normalmente. Debería ponerse en situación de juego, pero sin jugar y con supervisión externa del coterapeuta (madre o pareja). Se le recordó que estaba autoexcluida y, aunque quisiera, no podría jugar, de modo que el objetivo era notar la ansiedad que le producía la posibilidad de jugar y no jugar finalmente. Se le dio la instrucción de acabar el ejercicio una vez hubiera disminuido su ansiedad a cero, o cerca de cero, pero nunca antes.

Nivel 2: La pauta fue idéntica al primer nivel; sin embargo, se aumentó la frecuencia del ejercicio a dos veces por la mañana, momento en que la hija se encontraba en el colegio. Si 
todo iba bien, se le sugirió que pasara a realizar el ejercicio sola, sin presencia del coterapeuta. Se recordaron técnicas entrenadas y se elaboraron autoinstrucciones individualizadas para poner en práctica durante el ejercicio. Se explicó que si en algún momento se sentía superada, debería volver al nivel anterior y llamar a la terapeuta.

Nivel 3: Este último nivel consistió en complicar el ejercicio de forma que fuera lo más parecido a su conducta habitual de juego. La instrucción fue la misma que en niveles anteriores, pero con una diferenciación clave: realizaría el ejercicio desde su smartphone, tal y como solía jugar a video bingo. Debería hacerlo varias veces por la mañana y una por la tarde, sentada y sin supervisión del coterapeuta. Se le instruyó para que retrocediera al nivel anterior y realizara el ejercicio con la tablet en caso de que no fuera capaz de disminuir su ansiedad. Si iba bien, una vez disminuida la ansiedad, debería cortar el ejercicio y ponerse a ver un capítulo de su serie preferida. El objetivo final era aprender a parar y retomar el control.

Tabla 3: Ejemplo de registro de exposición

\begin{tabular}{|c|c|c|c|c|c|c|c|}
\hline & Lunes & Martes & Miércoles & Jueves & Viernes & Sábado & Domingo \\
\hline Evento & Starvegas & Starvegas & Starvegas & Starvegas & Starvegas & Starvegas & Starvegas \\
\hline Deseo de apostar & -2 & -1 & -0 & -1 & -2 & -1 & -2 \\
\hline Ansiedad durante & -3 & -2 & -1 & -1 & -1 & -1 & -1 \\
\hline $\begin{array}{l}\text { Ansiedad al acabar } \\
(0-10)\end{array}$ & -0 & -0 & -0 & -0 & -0 & -1 & -0 \\
\hline Evento & Starvegas & Starvegas & Starvegas & Starvegas & Starvegas & Starvegas & Starvegas \\
\hline Deseo de apostar & -2 & -2 & -1 & -2 & -2 & -3 & -1 \\
\hline Ansiedad durante & -1 & -1 & -1 & -1 & -2 & -2 & -1 \\
\hline $\begin{array}{l}\text { Ansiedad al acabar } \\
(0-10)\end{array}$ & -0 & -1 & -0 & -0 & -0 & -0 & -0 \\
\hline
\end{tabular}

Fase 4. Prevención de recaídas. Se realizó la prevención de recaídas, tanto a través de sesiones individuales ( 3 sesiones) como en pequeños grupos de cinco ( 2 sesiones). El objetivo fue utilizar la dramatización y role-playing, así como el debate y el formato de grupo de discusión para aprender a analizar las recaídas ocurridas tanto en C. como en otros pacientes y obtener un aprendizaje en grupo. Dentro de este entrenamiento en prevención de recaídas se trabajó especialmente el reconocimiento de C. sobre cuáles eran sus situaciones de riesgo y cómo resolverlas anticipadamente con éxito: evitando el riesgo y reaccionando apropiadamente sobre la situación. Las tareas para casa fueron el registro de visibilización de recaídas y registro de metas y valores con el que se pretendió que $\mathrm{C}$., una vez recordadas sus potencialidades y fortalezas, ordenara sus valores y estableciera objetivos a medio y largo plazo en consonancia con sus principios de vida.

A lo largo de todas y cada una de las fases descritas se intercalaron dos sesiones transversales de grupo donde se proporcionó información veraz sobre el problema de juego, el diseño 
de los tipos de juego a cargo de las empresas de juego online y el marketing y la publicidad que utilizan. El objetivo fue generar un cambio de actitudes de los participantes respecto al juego que les alejara de volver a jugar entendiendo que se trata de un negocio, pero un negocio para estas empresas y a costa del grave riesgo que supone para la salud de los jugadores (Chóliz, 2018).

\section{RESULTADOS}

\section{Patrón de juego}

La paciente dejó de jugar una vez comenzado el tratamiento y sigue sin jugar después de un periodo de seguimiento de tres meses.

C. tuvo dos caídas a lo largo del proceso terapéutico. La primera de ellas ocurrió en los momentos iniciales de la exposición y coincidiendo con cobrar el paro. El primer día perdió 200 euros y al día siguiente volvió a jugar y lo recuperó. El juego estuvo relacionado con errores en la forma de llevar a cabo la exposición y la presencia de páginas ilegales sobre las que no tiene eficacia el control de estímulo.

La segunda caída fue durante la fase de prevención de recaídas y fue como despecho hacia su compañero, que se había retirado a dormir cuando ella quería seguir divirtiéndose.

Criterios de juego patológico

Se evaluó el diagnóstico de juego patológico a través del NODS (Gerstein et al., 1989). Pasó de un diagnóstico de "trastorno de juego grave" (9 criterios del DSM-5) al principio del tratamiento, a ausencia de trastorno de juego (1 criterio del DSM-5) al finalizar la intervención.

\section{Sintomatología clínica}

Se evaluaron los síntomas clínicos y psicopatológicos con el SCL-90. Al inicio del tratamiento, C. presentaba un perfil más grave que el de la población clínica, mientras que después del tratamiento no solamente habían desaparecido los síntomas clínicos, sino que el

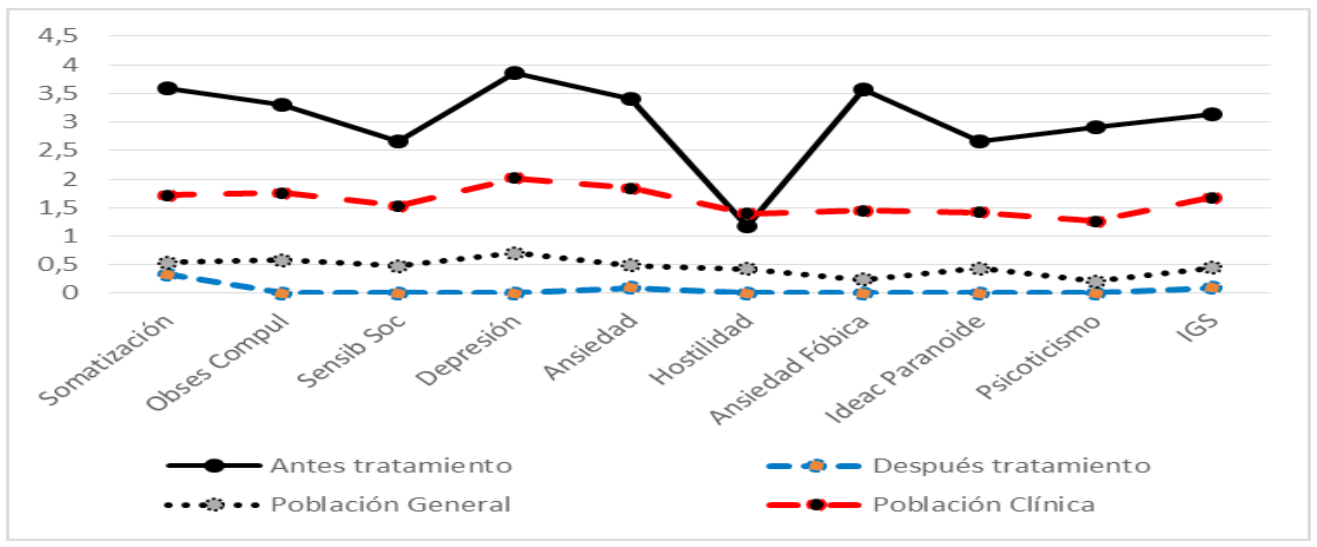

Figura 2- Sintomatología clínica antes y después del tratamiento. Comparación con muestra clínica y población general. 
perfil era más bajo que el de la población general en todas las dimensiones del SCL-90, tal y como se refleja en la Figura 5. Esta mejoría se generalizó a los principales aspectos de su vida y ella misma lo reconoció, tal y como se refleja en la carta que escribió a su terapeuta, que aparece en el Anexo de este artículo.

\section{Consumo de fármacos}

La paciente venía derivada de un servicio de salud mental por sus problemas de ansiedad, depresión e insomnio. La prescripción médica al inicio del tratamiento era un combinado de antidepresivos, neurolépticos, ansiolíticos e hipnóticos, en concreto: Pristiq (2-1-0), Apaloz (1-0-0), Tranquimazin (1-1-1) y Noctamid (0-0-1).

Se fue produciendo una reducción progresiva de los fármacos, comenzando con los hipnóticos prescritos para conciliar el sueño, seguido de las benzodiacepinas. Noctamid se le retiró tras el entrenamiento en relajación y las benzodiacepinas se pautaron en 9 semanas. Al finalizar el tratamiento no consumía fármacos y tiene pendiente una revisión en salud mental para la retirada de antidepresivos y neurolépticos.

Cambios en el estilo de vida

Durante la fase de control de estímulo y superación de la abstinencia se le propusieron las siguientes actividades diarias complementarias o incompatibles con el juego: a) paseos por la ciudad; b) implicación en la ayuda escolar de su hija; c) implicación en las actividades de ocio de su hija (salir al parque con ella). Además, se programaron actividades para el fin de semana.

Al finalizar el tratamiento, la mayoría de esas actividades se habían convertido en cotidianas, conformando un nuevo estilo de vida alternativo al del juego que tenía al iniciar el tratamiento, que era estar tumbada en la cama y pasar mucho tiempo en casa viendo la televisión o utilizando el móvil.

\section{CONCLUSIONES Y DISCUSIÓN}

A pesar de que el tratamiento está especialmente dirigido a la reducción de la conducta de jugar y fomento de un estilo de vida alternativo, tanto el cumplimiento de esos objetivos, como la aplicación de las técnicas necesarias para conseguirlos, redundaron en una mejoría global del estado de ánimo, su propia autoestima y sus relaciones sociales. Así, la aplicación de las técnicas de control de estímulo y de exposición le permitieron tener un control del juego que mejoraron no solamente su autoeficacia, sino su propia autoestima. Igualmente, las sesiones de grupo e individuales sobre juego ético le ayudaron a cambiar la concepción del juego y las actitudes hacia el mismo, lo cual le sirvió para mantener el necesario esfuerzo y constancia cuando se encontraba en momentos difíciles durante el proceso terapéutico. Pero, además, con la aplicación de las técnicas de relajación y control de la ansiedad que se utilizaron para la superación del malestar o el craving de juego, C. fue reduciendo el consumo de ansiolíticos e hipnóticos y el psiquiatra le pautó una retirada progresiva del resto de fármacos. Asimismo, la práctica de actividades de ocupación del tiempo libre, necesarias para la reducción del tiempo y momentos dedicados a jugar, le supuso ampliar el repertorio conductual con comportamientos alternativos e incompatibles con el juego. Finalmente, la 
implicación en actividades escolares y relacionales con su hija, como alternativa a tumbarse y jugar a video bingo favoreció el que ampliara objetivos e intereses vitales.

Todo ello redundó en una mejoría general de su salud mental, lo cual se reflejaba tanto subjetivamente (manifestaciones en las sesiones y en la carta enviada a la terapeuta), como objetivamente en las puntuaciones obtenidas en el SCL-90, que pasó de tener un perfil muy grave a ser inferior al de la población general.

Las dos caídas que la paciente tuvo a lo largo del proceso terapéutico fueron aprovechadas para entrenar en prevención de recaídas y consolidar todavía más los cambios. La primera de ellas ocurrió en los momentos iniciales de la exposición y concurrieron varios fenómenos. Por un lado, un incumplimiento de las condiciones en las cuales se debía haberse sometido a la extinción. Y por otra, un evento que ni siquiera sospechaban los terapeutas que anuló el efecto de la autoexclusión.

Así, durante las primeras sesiones de exposición, la paciente debía exponerse de forma controlada mediante una tablet sentada en el sillón de su casa y navegando en páginas de video bingo, pero sin entrar en ellas. Las condiciones eran bastante favorables al control, puesto que nunca le había gustado jugar con la tablet, ya que funcionaba peor que el ordenador, y además lo hacía sentada en el sillón, cuando ella siempre jugaba en la cama. Además de ello, la paciente se había autoexcluido en la Dirección General de Ordenación de Juego, de manera que no era posible que jugara en páginas legales de juego.

Pese a ello, un día después de cobrar el paro, "sin saber cómo pasó”, jugó durante dos días. El primer día perdió doscientos euros y el segundo volvió a jugar y recuperó: "por lo menos no he perdido nada”, llegó a decir. Se aprovechó esta circunstancia para analizar la recaída y tomar las medidas adecuadas.

En cuanto al deseo de jugar, este se explicó porque se estaba exponiendo a situaciones de juego y ello le provoca la respuesta condicionada de abstinencia que debe extinguir. Además, había cobrado el paro, lo cual es una situación de riesgo que también favorece la aparición de dichas respuestas condicionadas. Se encontraba sola, sin actividad y, además incumplió algunas de las principales normas, como eran: a) usar la tablet, en lugar del móvil (ella dice que no le funcionaba); b) jugar en la cama, en lugar de en el sofá; y c) ir a otras páginas diferentes de las que se le había indicado, en concreto www.bingoolé.com, que es una página ilegal que, por lo tanto, permite jugar tanto a menores como a personas que se han autoexcluido y que, además, había guardado los datos bancarios de la paciente en ocasiones en las que había jugado en el pasado.

Se dedicó un tiempo a analizar estas causas y al entrenamiento de las estrategias que habrían hecho detener la cadena conductual, tales como: distracción, detención de pensamiento y realización de actividades alternativas. Se le repitió que, a pesar de que no es culpable de lo ocurrido, sí que es responsable de su recuperación y de las consecuencias de su conducta y que, por lo tanto, debía anular la cena que tenía para el fin de semana como recompensa de haber estado sin jugar durante el último mes, así como denunciar a la web ilegal de juego ante la DGOJ, para lo cual debería escribir una carta de puño y letra y enviarla por correo certificado. Se reforzó el control de las actividades alternativas y de las páginas de juego, 
las técnicas de reducción de la ansiedad (estaba muy alterada por lo ocurrido) y se siguieron planificando las sesiones siguientes.

Se le comentó que esto era un paso atrás pero "para tomar impulso", de manera que habría que aprender de esta “caída” para salir más fuerte y más convencida de que el camino exige esfuerzo pero es superable. Además, estas recaídas hacen replantear la necesidad de adelantar el entrenamiento en prevención de recaídas a fases más tempranas del tratamiento y no dejarlo para el final de la intervención.

A pesar de que la paciente ha logrado superar el problema de la adicción al juego, eliminado la conducta de juego y mejorado notablemente su ajuste emocional, la adaptación social, las relaciones familiares e interpersonales e incluso la actividad laboral, los terapeutas son cautelosos respecto a la evolución que pueda tener este caso. Preocupa que eventuales problemas laborales o de pareja puedan afectar a su estabilidad emocional y a la propia conducta de juego. Por ello se ha establecido un seguimiento más largo y a ser posible, una revisión anual a partir del segundo año de seguimiento, con el objetivo de estar atento a posibles recaídas. Además, la paciente tiene indicaciones de que puede realizar una llamada en cualquier momento y se le atenderá inmediatamente en la Unidad.

\section{ANEXO}

\section{Carta de la paciente a su terapeuta}

"El email lo envío desde el móvil, así que perdona faltas de ortografía porque quiero ir a toda leche y resulta más difícil.

Consegui el puesto de gestora de cobros. Conseguí un trabajo y lo dejé por éste; tuve que pasar dos entrevistas individuales, una dinámica grupal y un examen de mates. $Y$ ya he superado el periodo de prueba.

¡Yupiii!

Me encanta mi trabajo. Estoy aprendiendo una profesión nueva; según me comentaron, me contrataron porque me vieron potencial, que era una gran negociadora. Y se me da genial; lo que no saben es que yo he estado en el otro lado, como deudora. Tal vez por eso trato bien a la gente, intento encontrar soluciones a sus deudas ofreciéndoles pagos parciales $e$ incluso quitas. Mi supervisora no para de decirme que le encanta cómo trabajo y es súper cariñosa. Me abraza cuando hago algo que le ha encantado, escucha las llamadas que creo que son geniales para que me diga dónde puedo mejorar. Estoy encantadísima; cada día aprendo, ayudo a personas a encontrar la mejor solución a sus problemas de pago (la gran mayoría agradece mi llamada, mi atención, mis acuerdos... porque me pongo en su lugar), me valoran mis superiores y el trabajo me gusta.

Y ahora viene lo mejor...

Mi chico y yo nos hemos ido a vivir juntos. Así que me levanto antes para dejar a la niña en el cole, desayunamos en casa de mi madre para verla todos los días, la bajo al cole y yo me vengo a casa. Tengo pensado comer a diario en casa de mami pero aún tengo cosas que hacer en el piso, todavía tenemos cositas por ahí. Tendrías que verlo, es precioso. Y 
estamos súper a gusto aquí y me pilla a diez minutos del cole de la niña. Todo va genial, estamos más unidos desde que empezamos con la mudanza y todo eso, el sexo es diario y yo estoy encantada.

También voy andando al trabajo, así que entre los paseos para el cole y los del trabajo, hago ejercicio. He tenido que dejar el gym por falta de tiempo. Pero lo retomaré si puedo.

¡Ahhhh!, me he puesto a régimen. Buena alimentación y una hora de caminata al día surtirán efecto.

$Y$ claro te preguntarás: ¿por qué no me habla del juego? Porque ni tengo tiempo, ni ganas, ni me interesa. Ahora tengo un trabajo que me gusta y si se acaba buscaré otro, no me pienso deprimir porque tengo potencial. Y lo que me encanta es estar tan liada. No tengo tiempooooooo.

¿Te acuerdas de mi vida cuando fui en mayo?

Mírame ahora...

Muchos besotes guapísima y gracias por existir."

Abbott, M.W., Volberg, R.A., \& Ronnberg, S. (2004). Comparing the Swedish and New Zealand national surveys of gambling and problem gambling. Journal of Gambling Studies, 20 (3), 237-258.

APA, American Psychiatric Association (2013). Diagnostic and statistical manual of mental disorders (DSM-5). Washington, DC: American Psychiatric Pub.

Bakken, I.J., Götestam, K.G., Grawe, R.W., Wenzel, H.G., y Øren, A. (2009). Gambling behavior and gambling problems in Norway 2007. Scandinavian Journal of Psychology, 50, 333-339.

Barlow, D. H., y Hersen, M. (1998). Diseños experimentales de caso único. Barcelona: Martínez Roca.

Blanco C, Hasin DS, Petry N, Stinson FS, Grant BF. Sex differences in subclinical and DSM-IV pathological gambling: results from the National epidemiologic survey on alcohol and related conditions. Psychological Medicine, 36(7):943-53

Chóliz, M. (2016). The challenge of online gambling: the effect of legalization on the increase in online gambling addiction. Journal of Gambling Studies, 32(2), 749-756.

Chóliz, M. (2017). Ludens: programa de prevención de adicción al juego. Valencia: Psylicom.

Chóliz, M. (2018). Ethical gambling: a necessary new point of view of gambling in public health policies. Frontiers in Public Health, 6, article 12. doi: 10.3389/fpubh.2018.00012.

Chóliz, M. y Marcos, M. (en prensa). Tratamiento de la adicción al juego online: guía clínica. Madrid: Pirámide

Chóliz, M.; Marcos, M. y Lázaro-Mateo, J. (2019). The risk of online gambling. A study of gambling disorder prevalence rates in Spain. International Journal of Mental Health and Addiction. https:// doi.org/10.1007/s11469-019-00067-4

Derogatis, L. R. (1979). Symptom Checklist-90-Revised (SCL-90-R). Lyndhurst, NJ: NCS Pearson.

Echeburúa, E. y Fernández-Montalvo, J. (2005). Psychological treatment of slot-machine pathological gambling: New perspectives. Journal of Gambling Studies, 21(1), 21-26.

Gerstein, D., Hoffmann, J., Larison, C., Engelman, L., Murphy, S., Palmer, A.,...y Hill, M.A. (1999). Gambling impact and behavior study. Report to the National Gambling Impact Study Com- 
mission. National Opinion Research Center at the University of Chicago, Chicago, National Opinion Research Center.

Granero, R., Penelo, E., Martínez-Giménez, R., Álvarez-Moya, E., Gómez-Peña, M., Aymamí, M. N., ... y Jiménez-Murcia, S. (2009). Sex differences among treatment-seeking adult pathologic gamblers. Comprehensive psychiatry, 50(2), 173-180.

Griffiths, M. y Barnes, A. (2008). Internet gambling: An online empirical study among student gamblers. International Journal of Mental Health and Addiction, 6, 194-204.

Griffiths, M., Wardle, H., Orford, J., Sproston, K. y Erens, B. (2009). Sociodemographic correlates of internet gambling: Findings from the 2007 British Gambling Prevalence Survey. Cyberpsychology \& Behaviour, 12, 199-202.

Johansson, A., Grant, J.E., Kim, S.W., Odlaug, B.L y Gotestam, K.G. (2009). Risk factors for problematic gambling: a critical literature review. Journal of Gambling Studies, 25, 67-92.

Miller, W. y Rollnick, S. (1991). La entrevista motivacional. Preparar para el cambio de conductas adictivas. Barcelona: Ediciones Paidós Ibérica, S.A.

Montero, I. y León, O. G. (2005). Sistema de clasificación del método en los informes de investigación en Psicología. International Journal of clinical and health psychology, 5(1), 115-127.

O 'Neill, R. E., Homer, R. H., Albin, R. W., Storey, K., y Sprague, J. R. (1990). Functional Analysis of Problem Behavior: A Practical Assesment Guide. Sycamore IL: Sycamore Publishing Company.

Volberg, R. (2001). When the chips are down: Problem gambling in America. NY: A Century Foundation Report.

Wardle, H., Sproston, K., Orford, J., Erens, B., Griffiths, M., Constantine, R. et al. (2007). British Gambling Prevalence Survey. London, UK: National Centre for Social Research.

Welte, J.W., Barnes, G.M., Wieczorek, W.F., Tidwell, M.C. y Parker, J. (2002). Gambling participation in the U.S. - Results from a national survey. Journal of Gambling Studies, 18, 313-333.

Este trabajo ha sido posible en parte por la subvención del PNSD concedida a UNAD para el desarrollo desde la Universidad de Valencia de un protocolo de tratamiento psicológico de la adicción al juego online, en el marco de las subvenciones de 2017 del Ministerio de Sanidad para Fines de Interés Social. El protocolo de tratamiento de la Universidad de Valencia ha tenido en cuenta la perspectiva de género. 